\title{
Natural Blue Pigment from Gardenia jasminoides Ellis (Rubiaceae) as a Dental Plaque Disclosant
}

\author{
Myoung-Hee Kim¹, Min-Ho Lee ${ }^{2}$, and Young Sun Hwang ${ }^{1, \dagger}$ \\ 'Department of Dental Hygiene, College of Health Science, Eulji University, Seongnam 13135, ${ }^{2}$ Department of \\ Food Technology and Services, College of Health Industry, Eulji University, Seongnam 13135, Korea
}

\begin{abstract}
Background: Dental caries and periodontal disease are bacterial infectious disease, mainly caused by plaque, a bacterial colony deposited on the tooth surface and gum tissue. Dental plaque disclosants easily stain the dental plaque, making them effective for scaling and tooth brushing education. As the erythrosine typically contained in dental plaque disclosants is highly cytotoxic, a low toxicity additive is needed. In this study, we aimed to examine the natural pigments with negligible cytotoxicity but can effectively stain the dental plaques for use in dental plaque disclosants.

Methods: The pigmentation of eight types of natural pigments was tested on bovine tongue and teeth, as well as on head and neck tissue sections of experimental ICR mice. The cytotoxicity of gingival epithelial cells was measured via MTT assay. Pigmentation was performed on the bovine tongue and tooth surface. Pigmentation in the oral environment was observed in four mandibular incisors. A 2 Tone was used as a control.

Results: Of the eight types of natural pigments, purple and blue pigments were effective in coloring dental plaques on the enamel surface as well as in the head and neck tissue sections. Additionally, purple and blue pigments were visible on the surface of the bovine tongue. Red, pink, orange, green, purple, and yellow pigments showed strong cytotoxicity, whereas brown and blue pigments had relatively low cytotoxicity. Blue pigment was effective in staining the dental plaque of four mandibular incisors. Conclusion: We suggest that the blue pigment derived from Gardenia jasminoides Ellis (Rubiaceae), which is effective for coloring dental plaques and has low cytotoxicity, is useful as a naturally derived dental disclosant.
\end{abstract}

Key Words: Dental caries, Dental plaque, Oral hygiene, Periodontitis, Pigmentation

\section{Introduction}

Dental plaque is created by bacterial colonies that are difficult to remove by general oral cleaning and are intimately related to both dental caries and gum disease ${ }^{1)}$. Therefore, proper removal of dental plaque from the tooth surface is vital for maintaining oral health. In general, dental plaques are transparent, colorless, and difficult to identify, meaning that individual plaque management has limitations. The use of dental disclosants to identify the degree and location of dental plaques facilitates dental plaque management and contributes to oral disease prevention. In particular, dental disclosants are very helpful in teaching children proper oral health techniques, as the brightly colored plaque clearly shows them the areas they are failing to clean ${ }^{2}$.

The tar pigment erythrosine, otherwise known as Red No.3, is an organo-iodine compound used as a component in dental disclosants ${ }^{3)}$. To improve the contrast between the gingival tissue and the stained dental plaque, a 2 Tone dental disclosant in which erythrosine is mixed with Fast Green and Brilliant Blue is commonly used ${ }^{4)}$. The 2 Tone dental disclosants stain common plaques in red, while dental plaques that have accumulated low $\mathrm{pH}$ bacteria for 
a long period of time are stained blue. According to a 1986 report, dental disclosants containing erythrosine showed high cytotoxicity in cultured human cells ${ }^{5}$. In experimental mice fed with high doses of erythrosine, thyroid cancer formation was induced by chronic thyroid stimulation via thyroid-stimulating hormone ${ }^{6}$. In addition, erythrosine causes a serotonin secretion disorder, resulting in depression and personality disorders ${ }^{7}$. In 1990, the FDA (U.S. Food and Drug Administration) banned the use of erythrosine, but many countries around the world, including Korea and United States, still use dental disclosants containing erythrosine.

Although dental disclosants make it easy to distinguish dental plaques, persistent staining is not desirable. The 2 Tone dental disclosants have excellent dental plaque pigmentation but excessively persist, do not easily distain, and exhibit strong cytotoxicity. Given these facts, dental disclosants containing safe ingredients that stain well and wash away easily must be developed. In this study, natural pigments were trialed as components for dental disclosants that have low cytotoxicity and vibrant plaque staining. For this, the degree of coloration produced by natural pigments was determined using animal tissues. In addition, plaque coloration by natural pigments was compared to the 2 Tone dental disclosant on four mandibular incisors.

\section{Materials and Methods}

\section{Natural pigments}

The natural pigments used in the experiment were manufactured by Nafarm Co. Ltd. (Seongnam, Korea). The eight natural pigments included red (mixed Monascus red and carmine), pink (mixed cochinal extract, carmine, and Gardenia yellow), orange (mixed Monascus red and Gardenia yellow), green (mixed Gardenia yellow and Gardenia blue), purple (mixed Gardenia red and Gardenia blue), brown (caramel), yellow (Gardenia yellow), blue pigments (Gardenia blue). As a control, 2 Tone (Young Dental Manufacturing, Earth City, MO, USA) was used.

\section{Coloring test of natural pigments on bovine tongue and teeth}

Bovine tongue and permanent incisors with a healthy enamel surface were used on the day of slaughter. To test tongue staining, $20 \mu \mathrm{l}$ of the natural pigment solution was dropped on the surface, left for 1 minute, and washed three times with saline. To stain the enamel surface, a cotton swab was moistened with natural pigments and smeared evenly onto the surface, allowed to sit for 1 minute, and then washed three times with saline. The relative degree of pigmentation was analyzed using the Image $\mathbf{J}$ program in the same pixel area (National Institutes of Health, Bethesda, MD, USA).

\section{Tissue staining with natural pigments}

The head and neck tissue of 5-week-old ICR mice were embedded in paraffin and cut into $4 \mathrm{~mm}$ sections. After removing paraffin using xylene and $100 \% \sim 70 \%$ alcohol, 2 Tone and natural pigments were added to the tissue sections for 1 minute before washing the sections with phosphate buffered saline solution ( $\mathrm{pH}$ 7.2) three times. Hematoxylin \& Eosin (H\&E) staining was used as a control.

\section{MTT assay}

Gingival epithelial cells were obtained from Dr. Xianglan Zhang (Yonsei University College of Dentistry, Seoul, Korea) in passages $8 \sim 12$ and were cultured in Dulbecco's Modified Eagle Medium with Nutrient Mixture F-12 (3:1 ratio) (Gibco BRL, Gaithersburg, MD, USA), which contained $10 \%$ fetal bovine serum in a $37^{\circ} \mathrm{C}$ $5 \% \mathrm{CO}_{2}$ incubator. To test for cytotoxicity, the cells $\left(5 \times 10^{3}\right.$ cells/well) were cultured in media with 2 Tone or natural pigments in a 96-well plate for 24 hours. After replacing the media with $5 \mu \mathrm{g} / \mathrm{ml}$ MTT 3-(4,5Dimethylthiazol-2-yl)-2,5-diphenyl tetrazolium bromide (MTT), the cells were further cultured for 1 hour at $37^{\circ} \mathrm{C}$. Then $200 \mu \mathrm{l} /$ well dimethyl sulfoxide (Sigma-Aldrich, Temecula, CA, USA) was added, and formazan formation was estimated using a microplate reader (Synergy ${ }^{\mathrm{TM}}$ HTX Multi-Mode Microplate Reader; BioTek Instruments, Inc., Winooski, VT, USA) at $570 \mathrm{~nm}$.

\section{Coloring tests of natural pigments on mandibular incisors}

A cotton swab was moistened with 2 Tone or natural 
pigments and smear evenly on the four mandibular incisors of the participant ( $n=6 /$ group), left for 10 seconds, and washed twice with water. The area was photographed, and the relative coloring was measured in an equal pixel area using Image $\mathbf{J}$ program.

\section{Statistics}

Statistical analyses were conducted using InStat GraphPad Prism ver. 5.01 Statistical Software (GraphPad Software, Inc., San Diego, CA, USA). The results are expressed as mean \pm standard error. The experiments were repeated three times, and representative results were shown for each experiment. Non-parametric KruskalWallis test with Dunn's post hoc analysis was employed for multiple comparisons. Non-parametric Wilcoxon's signed rank test was used to compare the results of coloring tests of natural pigments on mandibular incisors. A p-value of $<0.05$ was considered significant.

\section{Results}

\section{Coloring test of natural pigments on bovine tongue and teeth}

The natural pigment solution was dropped on the surface of a bovine tongue, and the degree of staining was observed after washing the area with saline. Results showed that the degree of staining achieved by red, pink, orange, green, brown, and yellow pigments was relatively lower than that achieved by purple and blue pigments, likely because the contrast between the pigments and
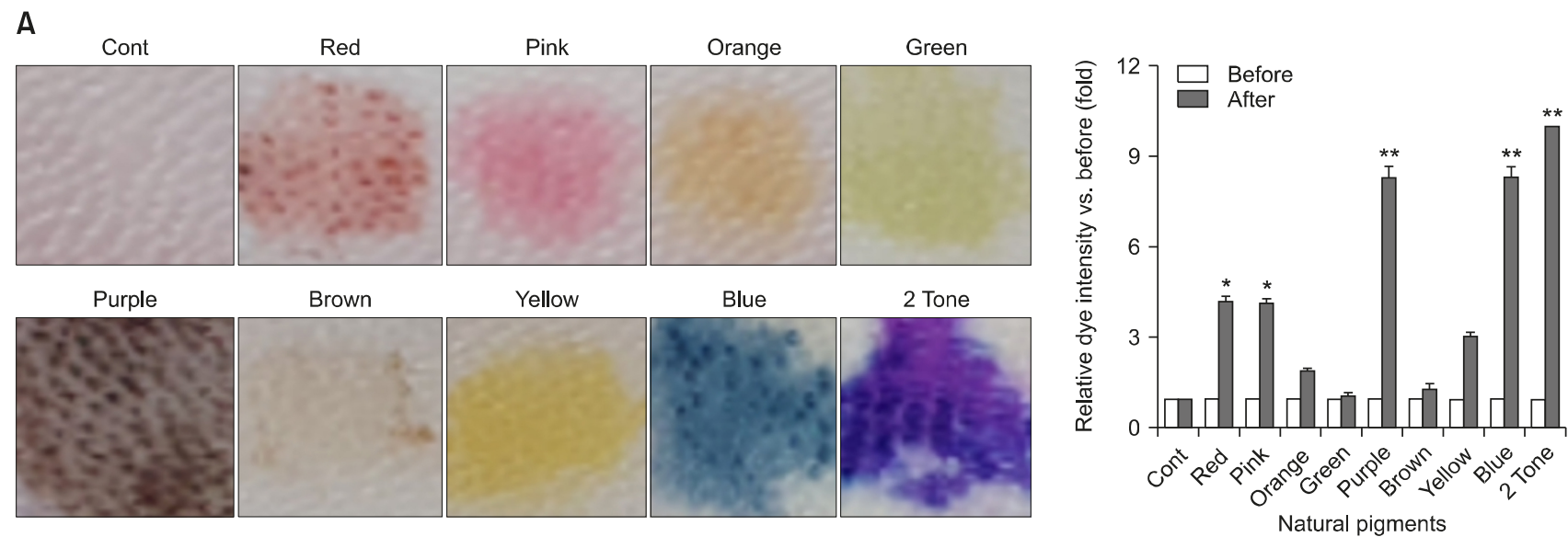

B
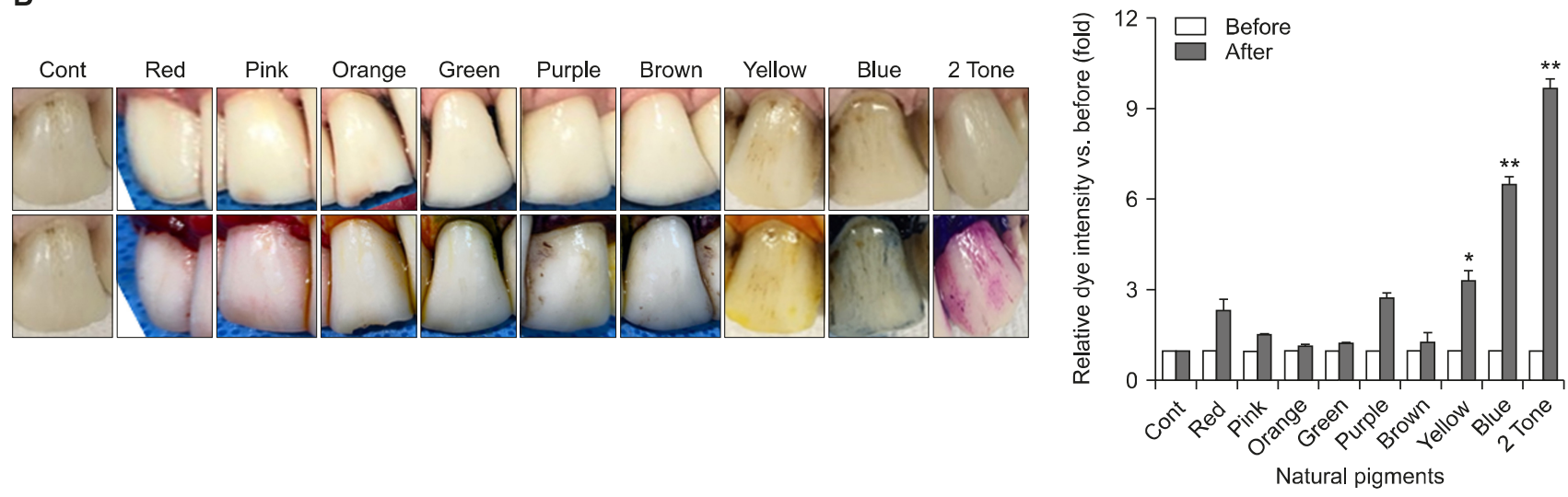

Fig. 1. Coloring tests of natural pigments on bovine tongue and teeth. (A) Equal amounts $(20 \mu \mathrm{l})$ of natural colorants were dropped on the surface of bovine tongue and left for 1 minute. After washing with saline, coloring was measured in equal pixel area using Image $\mathrm{J}$ program. The relative dye intensity before and after the experiment was plotted as a graph. ${ }^{*} \mathrm{p}<0.05,{ }^{* *} \mathrm{p}<0.001$ versus before. Dental disclosing solution 2 Tone was used as control. (B) A natural pigment solution was smeared on the enamel surface and washed with saline. Images were captured with a digital camera. Relative dye intensity before and after the experiment was plotted as a graph. ${ }^{*} p<0.05,{ }^{* *} p<0.001$ versus before. 
tongue surface color was not great (Fig. 1A). The coloration of the tongue surface by purple and blue pigments was similar to that of the 2 Tone. Purple and blue pigments were also effective in the coloring test on bovine tooth surfaces (Fig. 1B); however, the degree of staining was less than that of 2 Tone.

\section{Tissue staining with natural pigments}

Staining with natural pigments was observed after deparaffinizing the murine head and neck tissue sections. Compared with H\&E staining, the blue pigment widely stained the nucleus and cytoplasm of the bone, fiber, muscle, and blood cells. The degree of tissue staining achieved by red, pink, orange, green, purple, brown, and yellow pigments was low. The degree of staining by 2 Tone was similar to that of H\&E staining (Fig. 2).

\section{Cytotoxicity tests with natural pigments}

The cytotoxicity of 2 Tone has been previously reported ${ }^{5,8)}$. Cytotoxicity by natural pigments was tested using the MTT assays. As shown in Fig. 3, the red pigment showed strong cytotoxicity. For pink, orange, green, purple, and yellow pigments, excluding brown and blue pigments, relatively high toxicity was observed as the dilution decreased.

\section{Coloring tests of natural pigments on mandibular incisors}

According to the coloration and cytotoxicity results, of the eight natural pigments, the blue pigments were most effective in staining tissues and showed low cytotoxicity; therefore, blue pigment was chosen for further testing to observe the staining of mandibular incisors with frequent plaque formation. During the experiments, subjective evaluation revealed an unpleasant sour taste in 2 Tone and no taste in the blue pigment. Aanalysis of the degree of staining of the four mandibular incisors using the Image $\mathbf{J}$ program showed that the blue pigment was effective at staining the tooth surface compared with the 2 Tone $(\mathrm{p}=0.028)$ (Fig. 4).

\section{Discussion}

According to the healthcare big data open system from the Health Insurance Review and Assessment Service in

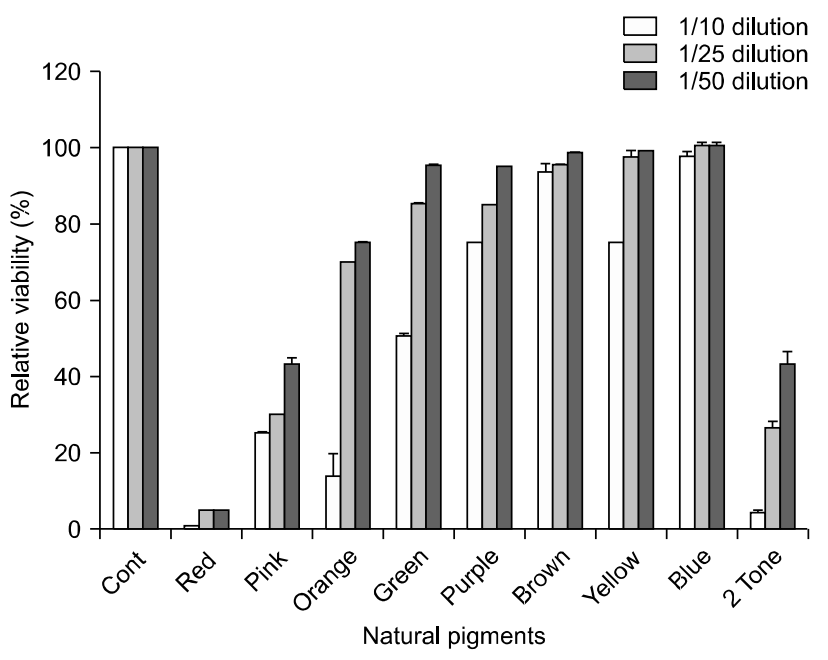

Fig. 3. Effect of natural pigments on cell viability. Gingival epithelial cells were cultured in the presence or absence of natural pigments at the indicated dilutions for 24 hours, and an MTT assay was performed. The values of the individual experiments are expressed as the mean \pm standard error of three independent experiments.

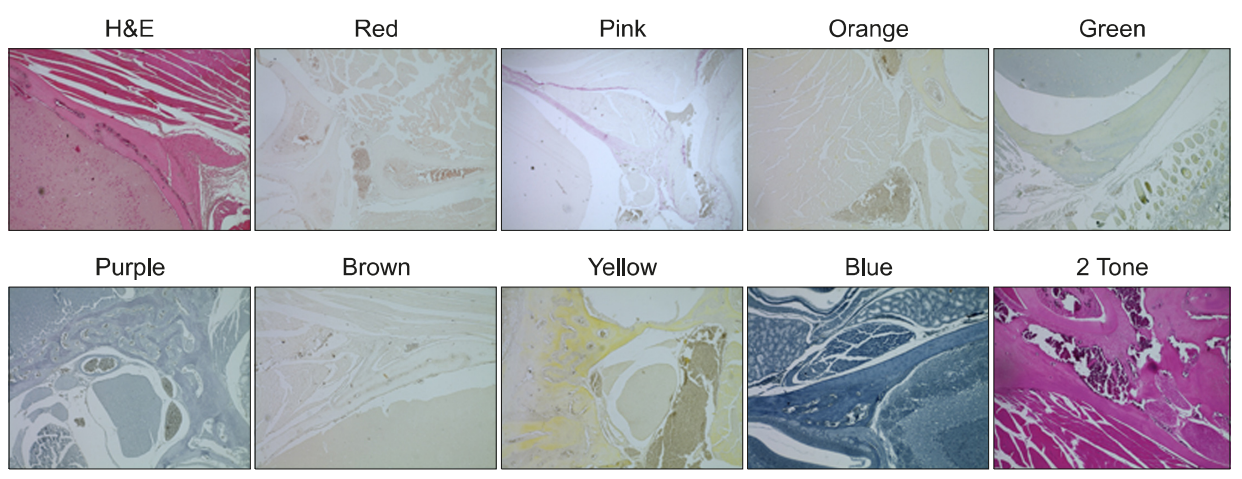

Fig. 2. Tissue staining with natural pigments. Deparaffinized mouse head and neck tissues were stained with natural pigments, hematoxylin \& eosin (H\&E), or dental disclosing solution 2 Tone (40x magnification). The slides were mounted and images were taken using a phasecontract microscope. 

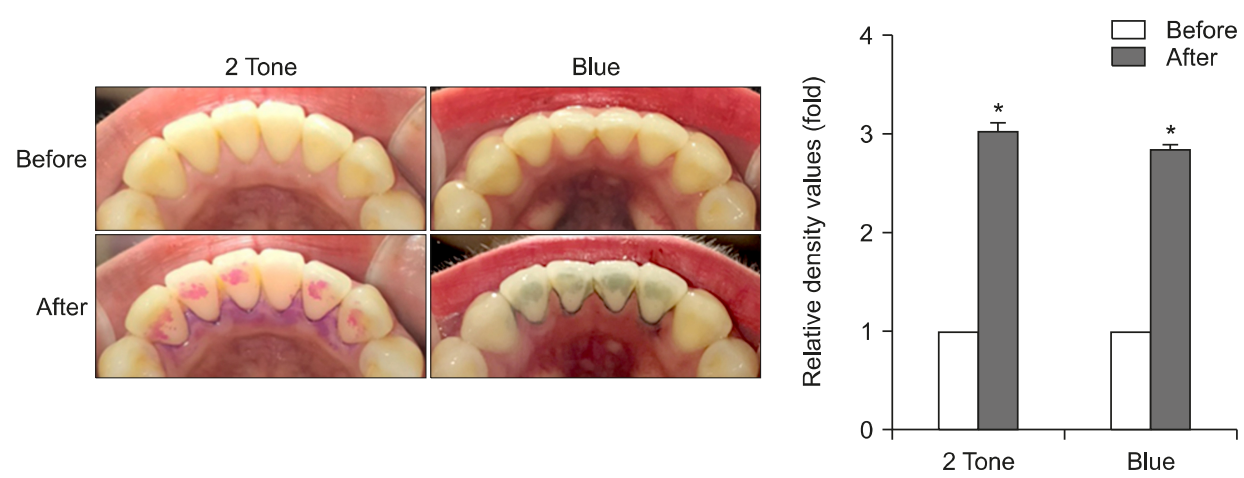

Fig. 4. Coloring tests of natural pigment on 4 mandibular incisors. A natural pigment solution was smeared on the enamel surfaces and left for 10 seconds. After washing with drinkable water, images were captured, and coloring was measured in equal pixel area using Image J program. The relative dye intensity before and after the experiment was plotted as a graph. The values of the individual experiments are expressed as the mean \pm standard error of three independent experiments. ${ }^{*} p<0.001$ versus before.

Korea, gingivitis and periodontal disease (K05), which has remained the second most frequent outpatient disease for the past 10 years, ranked first in both the total number of patients and the total amount of insurance payments, with 1,673 million patients and 15,321 billion won in medical care benefits reported in $2019^{9)}$. This is due to the high prevalence of gingivitis and periodontal disease, but it reflects an overall increased interest in oral health. Global epidemiology and search trends for oral problems using Google Trends clearly demonstrate that the prevalence of periodontal diseases is high and steadily increasing, making it the most common disease affecting humans worldwide $^{10,11)}$. Dental caries and periodontal disease are bacterial infectious disease, mainly caused by plaque, a bacterial colony deposited on the tooth surface and gum tissue. Therefore, plaque removal is very important for oral health management.

Dental disclosants stain plaque on enamel surfaces to help identify the degree and location of plaques, making it useful not only for professional dental treatment but also for personal oral care. Oral management education using dental disclosants is effective in promoting proper tooth brushing and oral hygiene due to its vibrant visual effect ${ }^{12)}$, and helps prevent dental caries and periodontal diseases $^{12,13)}$. If liquid dental disclosants are washed after application, unnecessary staining can be controlled, but the quick dissolving tablet causes excessive staining in oral cavity. Dental disclosants containing erythrosine are highly cytotoxic, meaning that lasting coloration is likely harmful to oral health ${ }^{8}$. In the head and neck tissues of experimental mice, dental disclosants containing erythrosine widely stained soft and hard tissues, but were difficult to wash away even with absolute alcohol ${ }^{8)}$. Using cell cycle and TUNNEL analyses in gingival epithelial cells, the cytotoxicity caused by dental disclosants containing erythrosine was confirmed to be due to apoptosis ${ }^{8)}$. As the carcinogenic effect of erythrosine has been clearly reported, use of substitute components has been suggested ${ }^{12)}$. Among the food pigments, Red No.40 (Allura Red, Red No.40), Blue No.1 (Brilliant Blue, Blue No.1), and mixed green (Yellow No.4 and Blue No.1) were assessed in antibacterial and panel tests; although biocompatibility was observed in all three, none have seen practical use so far.

In this study, an alternative dental disclosant ingredient with low cytotoxicity derived from natural pigments used for food was examined. Natural pigments included hongguk pigment (Monascus red), carmine pigment, cochineal extract pigment (podwarm rottenworm, quinoid type), caramel pigment, carthamus yellow pigments, Gardenia red pigments, and Gardenia blue pigment (fruit of the madder family Gardenia). As shown in the coloration and cytotoxicity test results, the blue pigment composed of Gardenia blue had the lowest cytotoxicity and highest effective plaque pigmentation when compared with the seven other pigments. Additionally, the blue pigment extensively stained not only the bone tissue, but also various soft tissues. In addition, the blue pigment stained similarly to the 2 Tone in the tests on four mandibular incisors. However, since the plaque pigmentation similar 
to that of blue pigment and 2 Tone is the result of comparing the staining degree before and after disclosure using the Image $\mathbf{J}$ program, a preference panel examination for the color contrast between gingival tissue and stained plaque will be required. Further analysis of the persistence of coloration of Gardenia blue pigment staining is also needed. Due to the coronavirus disease 2019 outbreak, it was difficult to recruit sufficient study participants and there were limitations on various intraoral experiments; these represent the major limitations but can be corrected after the pandemic has been brought under control.

Dental disclosants should selectively stain dental plaque, excluding gingival or other oral tissue, in order to be effective. In addition, the disclosant should not be harmful to oral health, should not exhibit prolonged pigmentation effects, and the color contrast between gingival tissue and stained plaque should be sufficiently high. Erythrosine exhibits an unpleasant taste and a non-selective pigmentation of oral tissues that is difficult remove. Erythrosine was banned in the United States because it causes autonomic nervous system disorders and carcinogenic effects. However, the Korea Food and Drug Administration only restricted its users in 2004 and still has not prohibied its use ${ }^{14)}$. In this study, we suggest that the blue pigment of Gardenia blue could be used as a substitute for erythrosine in dental disclosants. Gardenia blue pigment is a water-soluble pigment obtained by reacting $\beta$-glucosidase with iridoids glycosides in Gardenia jasminoides Ellis (Rubiaceae) and protein components ${ }^{15}$. Compared with the 2 Tone containing erythrosine, the plaque staining of Gardenia blue pigment was effective but not selective. However, the cytotoxicity and staining of gingival tissue by the Gardenia blue pigment were very low. During the experiment, the Gardenia blue pigment had largely no taste.

Since oral health is closely related to systemic health, proper oral care is very important, especially for toddlers, older adults, and patients with weakened immune system $^{16)}$. In order to continue the use dental disclosants for oral care in a safe and effective manner, we must determine the ingredients that have both selective plaque pigmentation and stability. From this study, we report that the Gardenia blue pigment is safe for gingival epithelial cells and that plaque pigmentation is sufficient to make Gardenia blue an effective dental disclosant. Therefore, the Gardenia blue pigment is a natural pigment that can replacing the cytotoxic erythrosine currently found in dental disclosants.

\section{Notes}

\section{Conflict of interest}

No potential conflict of interest relevant to this article was reported.

\section{Ethical approval}

Human experiment was approved by the Institutional Review Board (IRB) of the Eulji University (approval No. EU20-12).

\section{Author contributions}

Conceptualization: Min-Ho Lee and Young Sun Hwang. Experiments, Data acquisition: Myoung-Hee Kim, Min-Ho Lee, and Young Sun Hwang. Formal analysis: Myoung-Hee Kim, Min-Ho Lee, and Young Sun Hwang. Funding: Young Sun Hwang. Supervision: Myoung-Hee Kim and Young Sun Hwang. Writing-original draft: Young Sun Hwang. Writing-review \& editing: Young Sun Hwang.

\section{ORCID}

Myoung-Hee Kim, https://orcid.org/0000-0003-1589-4038

Min-Ho Lee, https://orcid.org/0000-0001-5464-8599

Young Sun Hwang, https://orcid.org/0000-0001-7012-3434

\section{Acknowledgements}

This research was supported by Basic Science Research Program through the National Research Foundation of Korea (NRF) funded by the Ministry of Education, Science and Technology (2018R1D1A1B07042035).

\section{References}

1. Kilian M, Chapple IL, Hannig M, et al.: The oral microbiome an update for oral healthcare professionals. Br Dent J 221: 
657-666, 2016.

https://doi.org/10.1038/sj.bdj.2016.865

2. Lee HR, Jung GU, Pang E: Comparison of the oral health education effect of air force personnel according to the use of disclosing solution. J Korean Dent Assoc 54: 57-66, 2016.

3. Carlson J: Selectivity of food colours for different organic acid transport systems in rat renal cortex. Acta Pharmacol Toxicol (Copenh) 41: 384-391, 1977. https://doi.org/10.1111/j.1600-0773.1977.tb02676.x

4. Datta D, Kumar SGR, Narayanan MBA, Selvamary AL, Sujatha A: Disclosing solutions used in dentistry. World J Pharm Res 6: 1648-1656, 2017. https://doi.org/10.20959/wjpr20176-8727

5. Watanabe E, Tsutsui T, Suzuki N, et al.: [Cytotoxicity of plaque-disclosing agents on cultured mammalian cells]. Shigaku 74: 1146-1152, 1986. Japanese.

6. Jennings AS, Schwartz SL, Balter NJ, Gardner D, Witorsch RJ: Effects of oral erythrosine (2',4',5',7'-tetraiodofluorescein) on the pituitary-thyroid axis in rats. Toxicol Appl Pharmacol 103: 549-556, 1990. https://doi.org/10.1016/0041-008x(90)90327-q

7. Yankell SL, Loux JJ: Acute toxicity testing of erythrosine and sodium fluorescein in mice and rats. J Periodontol 48: 228-231, 1977. https://doi.org/10.1902/jop.1977.48.4.228

8. Jung IH, Yeon KH, Song HR, Hwang YS: Cytotoxicity of dental disclosing solution on gingival epithelial cells in vitro. Clin Exp Dent Res 6: 669-676, 2020. https://doi.org/10.1002/cre2.321

9. Healthcare Bigdata Hub, Health Insurance Review and Assessment Service in Korea: Statistics of frequent disease in 2019. Retrieved December 24, 2020, from http://opendata.
hira.or.kr/op/opc/olapHifrqSickInfo.do.

10. Frencken JE, Sharma P, Stenhouse L, Green D, Laverty D, Dietrich T: Global epidemiology of dental caries and severe periodontitis - a comprehensive review. J Clin Periodontol 44 Suppl 18: S94-S105, 2017. https://doi.org/10.1111/jcpe.12677

11. Patthi B, Kumar JK, Singla A, et al.: Global search trends of oral problems using Google Trends from 2004 to 2016: an exploratory analysis. J Clin Diagn Res 11: ZC12-ZC16, 2017. https://doi.org/10.7860/JCDR/2017/26658.10564

12. Lee KH: Biocompatibility of food coloring agents to develop new dental plaque disclosants. J Korean Acad Pediatr Dent 26: 14-24, 1999.

13. Fasoulas A, Pavlidou E, Petridis D, Mantzorou M, Seroglou $\mathrm{K}$, Giaginis C: Detection of dental plaque with disclosing agents in the context of preventive oral hygiene training programs. Heliyon 5: e02064, 2019. https://doi.org/10.1016/j.heliyon.2019.e02064

14. National Institute of Food and Drug Safety Evaluation: Pharmaceutical additive guidelines - color additive status list. Retrieved December 25, 2020, from https://www.nifds.go. kr/brd/m_15/view.do?seq=10021(2016, December 20).

15. Ministry of Food and Drug Safety: Food and Food Additive Code. Gardenia blue. Retrieved December 25, 2020, from https://www.foodsafetykorea.go.kr/foodcode/04_03.jsp?idx $=443$.

16. Lertpimonchai A, Rattanasiri S, Arj-Ong Vallibhakara S, Attia J, Thakkinstian A: The association between oral hygiene and periodontitis: a systematic review and meta-analysis. Int Dent J 67: 332-343, 2017. https://doi.org/10.1111/idj.12317 